\title{
Modeling of the Turbulent Oil-Water Mixing Processes
}

\author{
Ivan V. Kazachkov ${ }^{1,2 *}$ \\ ${ }^{1}$ Dept of Information technology and data analysis, Nizhyn Gogol state university, Grafs'ka, 2, Ukraine \\ ${ }^{2}$ Dept of Energy Technology, Royal Institute of Technology, Sweden
}

*Corresponding Author: Ivan V. Kazachkov, Dept of Information technology and data analysis, Nizhyn Gogol state university, Grafs'ka, 2, Ukraine

\begin{abstract}
The mixing processes of the oil and water in different turbulent flows are important for optimal running of the technological devices and their optimal control. These classes of flow are multiphase because such liquids are mutually immiscible, which makes complication by their mathematical modeling, computer simulation and experimental study. The peculiarities of the turbulent two-phase flows of the oil-water as the mutually immiscible liquids are considered in this paper based on the method developed by Prof. Alfred I. Nakorchevskii. The method introduced the so-called function-indicators of the phases in a flow, which allowed recognizing the phases in their movement in a multiphase mixture, both theoretically and experimentally. The water jet spreading in the volume of oil and, inversely, oil jet spreading in the water are considered in the paper through the mathematical modeling and computer simulation.
\end{abstract}

Keywords: Turbulent Mixing; Numerical Simulation; Jets Oil-Water and Water-Oil; Immiscible Liquids.

\section{INTRODUCTION}

By the multiphase method of Prof. Alfred I. Nakorchevskii [1] the characteristics of mixture $a^{l}(t)$ (mass, velocity, impulse, etc.) of the corresponding different phases $a_{i}^{l}(t)$ in a multiphase flow are expressed as

$a^{l}(t)=\sum_{i=1}^{m} B_{i}(t) a_{i}^{l}(t)$

where $B_{i}(t)$ is co-called function-indicator determined as

$B_{i}(t)=\left\{\begin{array}{l}1, \text { if } i-\text { phase occupies the elementary volume } \delta \mathrm{V} \\ 0, \text { if } i \text { - phase is outside the elementary volume } \delta \mathrm{V}\end{array}\right.$.

Following to this approach, the analog of the Navier-Stokes equations in a boundary layer approximation is:

$$
\begin{aligned}
& \frac{\partial}{\partial x}\left(y \rho_{i} B_{i} u_{i}\right)+\frac{\partial}{\partial y}\left(y \rho_{i} B_{i} v_{i}\right)=0, \quad \sum_{i=1}^{m} B_{i}=1, \\
& \rho_{i} B_{i}\left(u_{i} \frac{\partial u_{i}}{\partial x}+v_{i} \frac{\partial u_{i}}{\partial y}\right)=-\frac{d p}{d x}+\frac{1}{y} \frac{\partial}{\partial y}\left[y B_{i} \tau_{i}\right]_{m},
\end{aligned}
$$

where is the sum by mute index $i$, which belong to a phase $i$. In the stationary equations of incompressible liquids (3) written in a cylindrical coordinate system are: $p$ - pressure, $\rho$ - density, $u, v$ the longitudinal and transversal velocity components, $\tau_{i}$ - turbulent stress for a phase $i$. Index $m$ belongs to the values at the axis of the flow (symmetry axis). All values are averaged on the given interval by time.

One liquid flow is going from the nozzle of the radius $r_{0}$ with velocity $u_{01}$ (the velocity profile is supposed uniform) in surroundings occupied by the other liquid (phase 2) being in a rest. The structure of a jets mixing with surrounding liquid is simplified according to the known scheme [2]. First, the 
initial part of the length $x_{\mathrm{i}}$ with the approximately linear boundaries for the conical surface (in cylindrical coordinates) of the internal potential core of a first phase and the external interface (conical too) are considered as the boundaries of the mixing area and the central potential core, correspondingly. The mixing turbulent zone between the above surfaces contains drops and fragments of the phases as far as immiscible liquids have behaviors like the separate phases, with their interfacial multiple surfaces interacting in all such locations (exchange of mass, impulse, energy among the phases). Close to the external interface, there is a flow of a surrounding liquid with some drops of the first phase, while, in turn, close to the boundary of the potential core there is a flow of the first phase with the drops of a second phase. As far as we have mutually immiscible liquids, the mixing zone contains two phase mixture in a turbulent flow. After an initial part of the mixing zone when all first phase in a potential core is spent, the short transit area is preparing the ground part of the turbulent two-phase jet, where both phases are well mixed across all layer. Usually the spatial averaging of the mass, momentum and energy conservation equations is performed using the concept of volumetric phase content for description of multiphase flows [3-6], which does not fit well to experimental study of the individual phase movement in a mixture. The method [1] was developed including the special experimental technology and micro sensor for the measurements in two-phase flows. In $[4,5]$ fundamentals and analysis of the different methods for modeling of the multiphase systems can be found. Actually, all known multiphase methods are well connected including [1], and the parameters averaged by time can be transformed to the ones averaged by space [3-6].

\subsection{Function-Indicator of the Phases in Two-Phase Flow}

External interface of the mixing zone is determined zero longitudinal velocity of the second phase and transversal velocity of the first phase (the second phase is injected from the immovable surrounding into the mixing zone). The function-indicator of the first phase $B_{1}(t)$ is zero at the external interface because the first phase is absent in surroundings. Similar, the function-indicator $B_{2}(t)$ is zero on the interface of the potential core, which is the boundary of the first phase (from the nozzle). The boundary conditions are [1]:

$y=y_{0}, \quad u_{i}=u_{0 i}, v_{i}=0, \tau_{i}=0, B_{1}=1 ; \quad y=y_{0}+\delta, u_{i}=0, v_{i}=0, \tau_{i}=0, B_{1}=0$.

The turbulent stress in the phases is stated by the "new" Prandtl's formula

$\tau_{i}=\rho_{i} \kappa_{i} \delta u_{m i} \partial u_{i} / \partial y$,

where $\kappa_{i}$ is the coefficient of turbulent mixing for $i$-th phase, $\delta$ is the width of the mixing layer.

\subsection{Polynomial Approximations of the Flow Velocities and Function-Indicator}

The polynomial approximations for the velocity profiles and other functions in the turbulent mixing zone have been obtained based on the boundary conditions (4) in the form:

$u_{1} / u_{01}=1-4 \eta^{3}+3 \eta^{4}$,

$u_{2} / u_{02}=1-6 \eta^{2}+8 \eta^{3}-3 \eta^{4}$,

$B_{1}=B_{1}^{(0)}=1-\eta^{3}+0.5 \eta^{2}(1-\eta) h(x), \quad h \in[-6,0]$,

$B_{1}=B_{1}^{(1)}=1-4 \eta^{3}+3 \eta^{4}+0.5 \eta^{2}(1-\eta)^{2} h(x), \quad h \in[-12,-6]$,

$B_{1}=B_{1}^{(2)}=1-10 \eta^{3}+15 \eta^{4}-6 \eta^{5}+0.5 \eta^{2}(1-\eta)^{3} h(x), \quad h \in[-20,-12]$,

where $h(x)=\left(\partial^{2} B_{1} / \partial \eta^{2}\right)_{\eta=0}$ - function determining a transition of the piecewise continuous functionindicator $B_{1}^{(n)}$ to its next approximation from the condition that derivative by $\eta$ at $\eta=1$ is zero up to $(n+1)$-th order.

\section{The Equations of Heterogeneous Two-Phase Jet at its Initial Part}

\subsection{Integral Correlations for the Initial Part of a Jet Flow}

The integral correlations were derived for the two-phase turbulent jet on the initial part as follows [1]: 


$$
\begin{aligned}
& u_{01}\left(r_{0}^{2}-y_{0}^{2}\right)=2 \delta \int_{0}^{1} B_{1} u_{1}\left(y_{0}+\delta \eta\right) d \eta \\
& \rho_{1} u_{01}^{2}\left(r_{0}^{2}-y_{0}^{2}\right)=2 \delta \int_{0}^{1}\left(\rho_{1} B_{1} u_{1}^{2}+\rho_{2} B_{2} u_{2}^{2}\right)\left(y_{0}+\delta \eta\right) d \eta \\
& \rho_{1} u_{01}\left(u_{01}-u_{1}^{*}\right) y_{0} y_{0}^{\prime}+\frac{d}{d x} \delta \int_{0}^{\eta^{*}} \sum_{j=1}^{2} \rho_{j} B_{j} u_{j}^{2}\left(y_{0}+\delta \eta\right) d \eta-\sum_{j=1}^{2} u_{i}^{*} \frac{d}{d x} \delta \int_{0}^{\eta^{*}} \rho_{i} B_{i} u_{i}\left(y_{0}+\delta \eta\right) d \eta= \\
& =\left(y_{0}+\delta \eta^{*}\right) \sum_{j=1}^{2} \rho_{j} B_{j} \kappa_{j} u_{0 j} \frac{\partial u_{j}^{*}}{\partial \eta}, \quad B_{1}+B_{2}=1 .
\end{aligned}
$$

Here the first equation (9) was got integrating by $y$ the mass conservation equation, the second and the third - integrating the momentum conservation for the total flow of two-phase mixture for $y=y_{0}+\delta$ and $y=y^{*}$, respectively. The polynomial approximations for the functions $u_{2}, B_{1}$ on a ground part of the jet keep the same but for the function $u_{1}$ approximation is as follows

$u_{1} / u_{m 1}=1-3 \eta^{2}+2 \eta^{3}$.

\subsection{Integral Correlations for the Ground Part of a Jet}

The integral correlations for the ground part of a jet flow obtained similarly to the above as follows [1]:

$$
\begin{aligned}
& 2 \int_{0}^{\delta} B_{1} u_{1} y d y=u_{01} r_{0}^{2}, \quad 2 \sum_{j=1}^{2} \int_{0}^{\delta} \rho_{j} B_{j} u_{j}^{2} y d y=\rho_{1} u_{01}^{2} r_{0}^{2}, \\
& \frac{d}{d x} \sum_{j=1}^{2} \int_{0}^{y^{*}} \rho_{j} B_{j} u_{j}^{2} y d y-\sum_{j=1}^{2} u_{j}^{*} \frac{d}{d x} \int_{0}^{y^{*}} \rho_{j} B_{j} u_{j} y d y=y^{*} \sum_{j=1}^{2} B_{j}^{*} \tau_{j}^{*},
\end{aligned}
$$

where the first is the equation of the mass conservation for the first phase, the second and the third the momentum conservation equations for the total and for the part of the cross section, respectively, according to the methodology [2]. And the momentum equation on the jet's axis $(y=0)$ is used too:

$$
\sum_{j=1}^{2} \rho_{j} B_{m j} u_{m j} \frac{d u_{m j}}{d x}=2 \sum_{j=1}^{2}\left[\frac{\partial}{\partial y}\left(B_{j} \tau_{j}\right)\right]_{m} .
$$

The mathematical model including the ordinary differential equations (9), (11), (12) by longitudinal coordinate $x$ is implemented for analysis and numerical simulation on the computer the basic features of the stationary turbulent two-phase jet of two immiscible liquids. The function-indicator $B_{1}$ shows how much is a presence of the first phase in a selected point of mixing zone. Solution of the task gives parameters of the flow together with their belonging to a particular phase.

\subsection{Dimensionless Form of the Outgoing Equation Array and the Profiles of Basic Parameters}

The equation array (9) with the boundary conditions (4) is used for numerical simulation of the turbulent two-phase jet on its initial part. For this, the equations (9) are transformed to the following dimensionless form with the scales $r_{0}, \delta, u_{0 i}$ for the longitudinal and transversal coordinates and velocity, respectively:

$$
\begin{aligned}
& y_{0}^{2}+2 \delta \sum_{j=1}^{2} y_{0}^{2-j} \delta^{j-1} a_{j}=1, \quad y_{0}^{2}+2 \delta \sum_{j=1}^{2} y_{0}^{2-j} \delta^{j-1}\left(a_{j+2}+i_{0} b_{j+2}\right)=1 \\
& \left(1-u_{1}^{*}\right) y_{0} \frac{d y_{0}}{d \varsigma}+\frac{d}{d \varsigma} \delta \sum_{j=1}^{2} y_{0}^{2-j} \delta^{j-1}\left(a_{j+2}^{*}+i_{0} b_{j+2}^{*}\right)-\frac{d}{d \varsigma} \delta \sum_{j=1}^{2} y_{0}^{2-j} \delta^{j-1}\left(a_{j}^{*} u_{1}^{*}+i_{0} b_{j}^{*} u_{2}^{*}\right)=\left(y_{0}+\delta \eta^{*}\right)= \\
& =\left(y_{0}+\delta \eta^{*}\right) \sum_{j=1}^{2} B_{j}^{*}\left(\frac{\partial u_{j}}{\partial \eta}\right)^{*}\left(i_{0} \kappa_{21}\right)^{j-1}, \quad B_{1}+B_{2}=1
\end{aligned}
$$


Here are:

$$
\begin{aligned}
& \bar{y}_{0}=y_{0} / r_{0}, \bar{\delta}=\delta / r_{0}, \eta=\left(y-y_{0}\right) / \delta, \bar{x}=x / r_{0}, \varsigma=\kappa_{1} \bar{x}, s_{0}=u_{02} / u_{01}, i_{0}=n s_{0}^{2}, \\
& n=\rho_{2} / \rho_{1}, \kappa_{21}=\kappa_{2} / \kappa_{1}, a_{i}=a_{i 1}+a_{i 2} h, b_{i}=b_{i 1}+b_{i 2} h, \\
& a_{i}=\int_{0}^{1} B_{1} \bar{u}_{1} \eta^{j-1} d \eta, b_{i}=\int_{0}^{1} B_{2} \bar{u}_{2} \eta^{j-1} d \eta(i=1,2) ; a_{i}=\int_{0}^{1} B_{1} \bar{u}_{1}^{2} \eta^{j-1} d \eta, b_{i}=\int_{0}^{1} B_{2} \bar{u}_{2}^{2} \eta^{j-1} d \eta \quad(i=3,4) ; j=1,2 .
\end{aligned}
$$

Except the above, for the dimensionless parameters, we retain the same notations as for the dimensional ones. Only here in (14) it is stated for clarification of the dimensionless notations.

The sliding factor $s_{0}$ is supposed to be constant and the values of the parameters at $\eta=\eta^{*}<1$ are signed with a star *. The system (13) must satisfy the following boundary conditions

$\zeta=0, \quad y_{0}=1, \delta=0 ; \quad \zeta=\zeta_{i}, y_{0}=0, \quad \delta=\delta_{i}$;

where $\zeta_{i}, \delta_{i}$ are the dimensionless length of a jet and its maximal radius (at the end of the initial part).

\section{Numerical Simulation of the Initial Part of the Two-Phase JeT}

\subsection{Basics of the Turbulent Heterogeneous Jet on Initial Part}

A solution of the boundary problem (13)-(15) allows obtaining the functions $u_{m l}(\zeta), B_{m l}(\zeta), \delta(\zeta)$ and $h(\zeta)$ by the modeling parameters $i_{0}, \kappa_{21}$. The system (13) contains two algebraic and one differential equation. From the algebraic equation array the functions $y_{0}(h), \delta(h)$ are got. And then the differential equation is solved numerically. The range of the function's $h(\zeta)$ variation is determined by substitution of the boundary conditions in the functions $y_{0}(h), \delta(h)$, so that we obtain the next:

$$
\begin{aligned}
& y_{0}=\frac{1}{\sqrt{1+2 a_{1} \frac{a_{3}+i_{0} b_{3}-a_{1}}{a_{2}-i_{0} b_{4}-a_{4}}+2 a_{2}\left(\frac{a_{3}+i_{0} b_{3}-a_{1}}{a_{2}-i_{0} b_{4}-a_{4}}\right)^{2}}, \quad \delta=y_{0} \frac{a_{3}+i_{0} b_{3}-a_{1}}{a_{2}-i_{0} b_{4}-a_{4}} ;} \\
& h_{0}=h(0)=\frac{a_{11}-a_{31}-i_{0} b_{31}}{a_{32}-a_{12}-i_{0} b_{32}}, \quad h_{i}=h\left(\varsigma_{i}\right)=\frac{a_{21}-a_{41}-i_{0} b_{41}}{a_{42}-a_{22}-i_{0} b_{42}} .
\end{aligned}
$$

\subsection{The Results of Computer Simulations for the Oil Jet Spreading in Water}

The results of numerical simulations are presented below. For $i_{0}=0.80$ (water jet spreading in oil pool), $\kappa_{21}=0.5$ the results are given in Figs 1-3 in two different scales to see clear the behaviors of the functions on their distance from the nozzle. It was noted that with increase of turbulent mixing coefficient the initial part of the jet is shortened. Here $\varsigma_{i}=\kappa_{1} \bar{x}=1.55$ in figs, afterwards it is not real, because the initial part of the jet is finished, $y_{0}=0, \delta=\delta_{i}$. If turbulent mixing coefficient for water is 100 times higher than the laminar one, $\kappa_{1}=0.1$, then $\varsigma_{i}=\kappa_{1} \bar{x}=1.55$ means $\bar{x}=15.5$, so that the initial part of the jet is just 15.5 radiuses of the jet by these parameters: $i_{0}=0.8, \kappa_{21}=0.5$. Then similar calculations are presented in Figs 4-6 for $\kappa_{21}=1.0$ and 5.0.
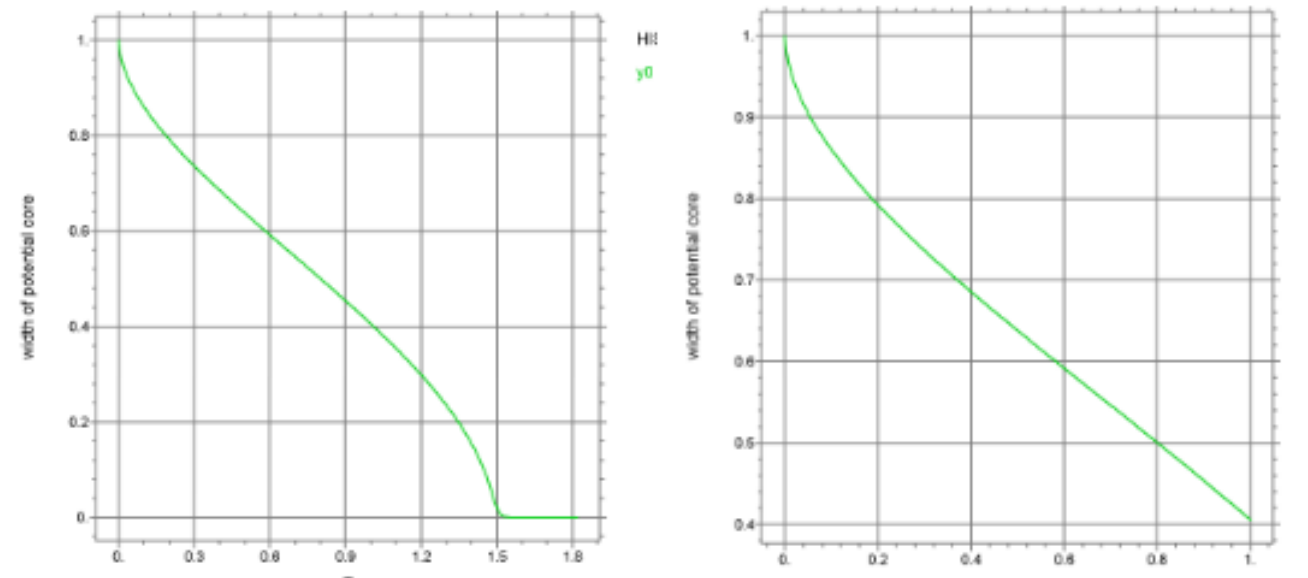

Fig1. The width of potential core $y_{0}(\zeta)$ in two different scales for parameters: $i_{0}=0.8, \kappa_{21}=0.5$ 

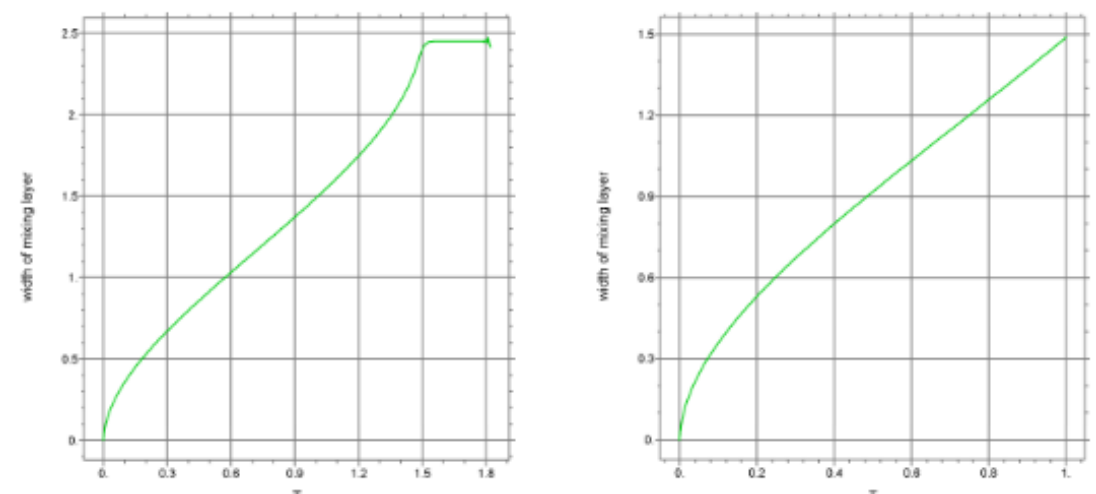

Fig2. The width of mixing layer $\delta(\zeta)$ in two different scales for parameters: $i_{0}=0.8, \kappa_{21}=0.5$
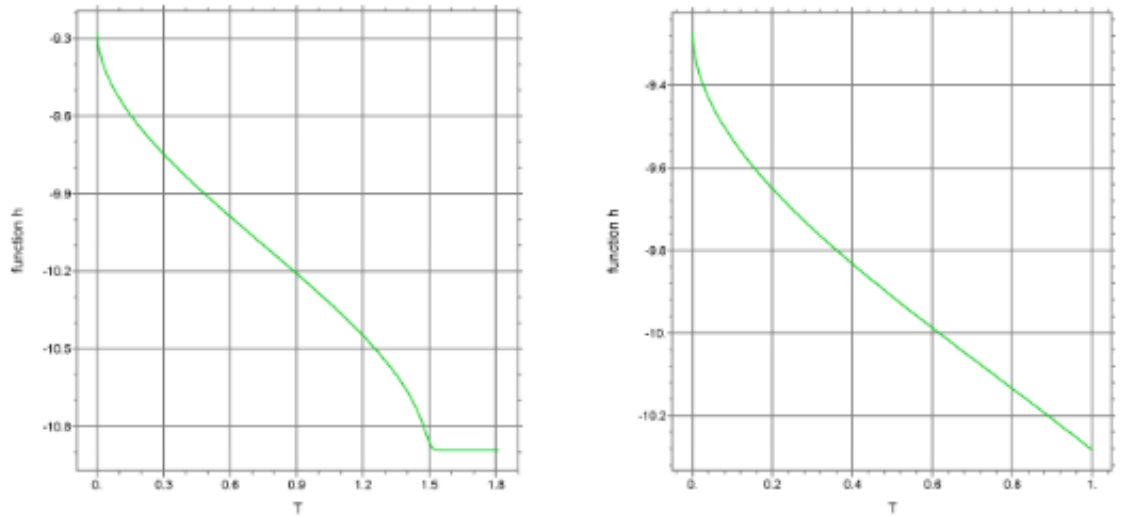

Fig3. The function $h(\zeta)$ in two different scales for parameters: $i_{0}=0.8, \kappa_{21}=0.5$

For $i_{0}=0.80, \kappa_{21}=1.0$ (with increase of turbulent mixing coefficient that initial part is shortened), $\varsigma_{i}=\kappa_{1} \bar{x}$ $=1.0$ in figs, afterwards it is not real, because the initial part of the jet is finished, $y_{0}=0, \delta=\delta_{i}$. If turbulent mixing coefficient for water is 100 times higher than the laminar one, $\kappa_{1}=0.1$, then $\varsigma_{i}=\kappa_{1} \bar{x}=1.0$ means $\bar{x}=10$, so that the initial part of the jet is just 10 radiuses of the jet by $i_{0}=0.8, \kappa_{21}=1.0$ (Figs 4-6):


Fig4. The width of potential core $y_{0}(\zeta)$ in two different scales for parameters: $i_{0}=0.8, \kappa_{21}=1.0$
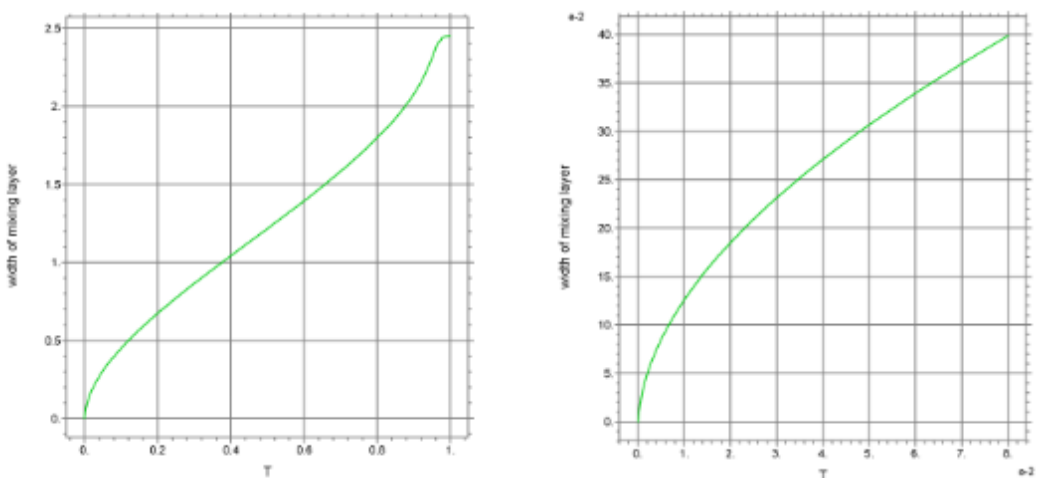

Fig5. The width of mixing layer $\delta(\zeta)$ in two different scales for parameters: $i_{0}=0.8, \kappa_{21}=1.0$ 

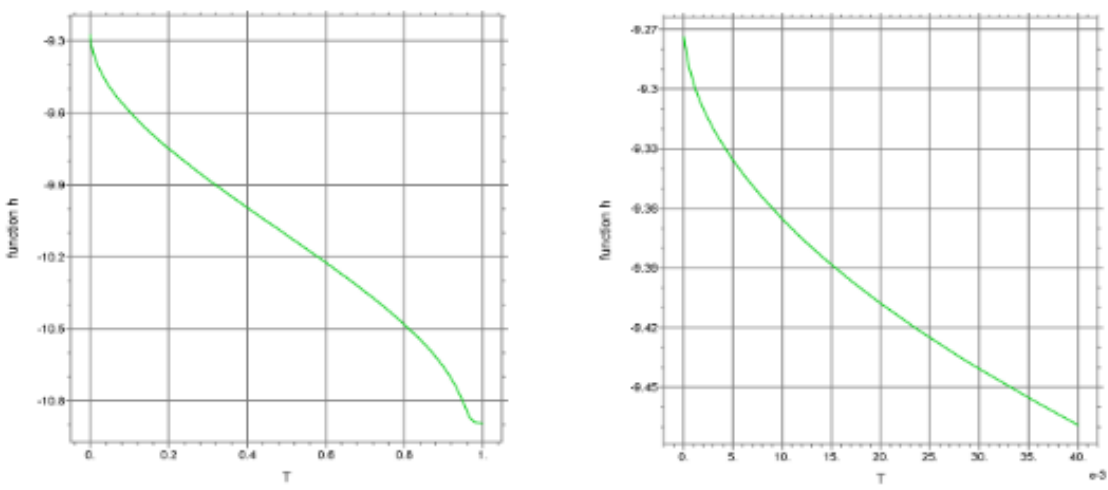

Fig6. The function $h(\zeta)$ in two different scales for parameters: $i_{0}=0.8, \kappa_{21}=1.0$

For $i_{0}=0.80, \kappa_{21}=5.0$ the results are presented in Figs 7-9, $\varsigma_{i}=\kappa_{1} \bar{x}=0.26$. If turbulent mixing coefficient for water is 100 times higher than the laminar one, $\kappa_{1}=0.1$, then $\varsigma_{i}=\kappa_{1} \bar{x}=0.26$ means $\bar{x}=2.6$ :


Fig7. The width of potential core $y_{0}(\zeta)$ in two different scales for parameters: $i_{0}=0.8, \kappa_{21}=5.0$
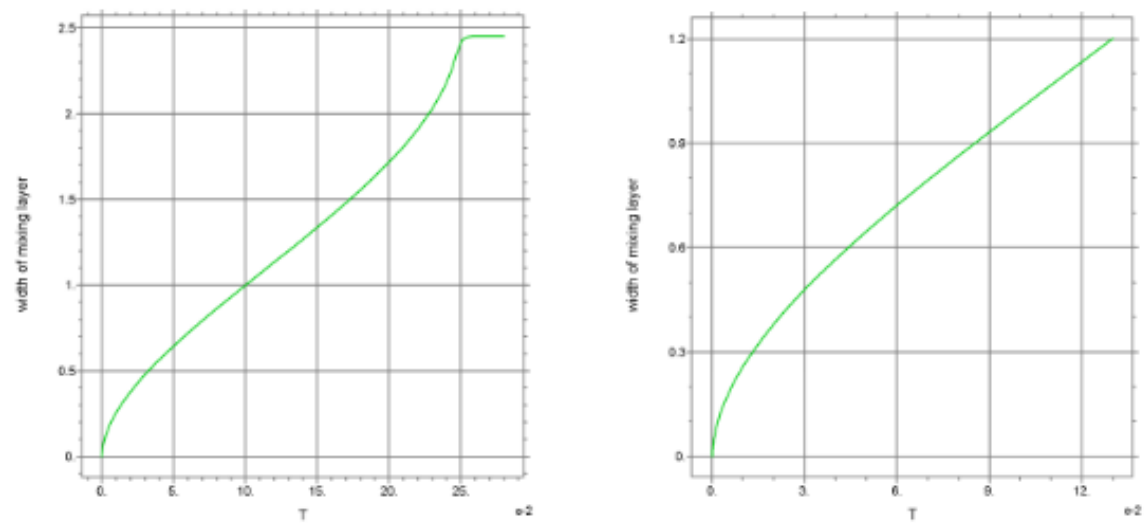

Fig8. The width of mixing layer $\delta(\zeta)$ in two different scales for parameters: $i_{0}=0.8, \kappa_{21}=5.0$
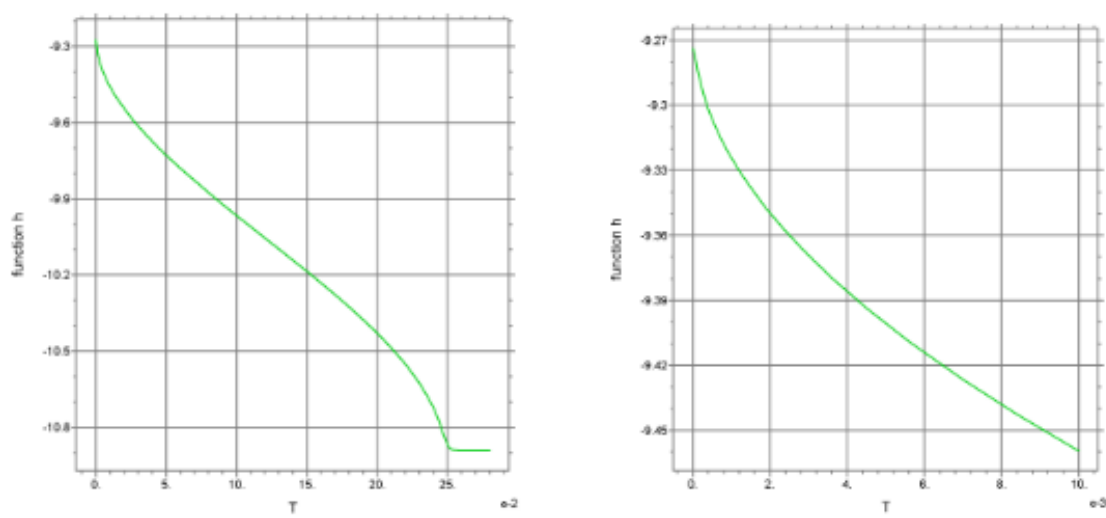

Fig9. The function $h(\zeta)$ in two different scales for parameters: $i_{0}=0.8, \kappa_{21}=5.0$ 


\subsection{The Results of Computer Simulations for the Water Jet Spreading in Oil}

For $i_{0}=1.2, \kappa_{21}=1.0$ the results of computer simulation are presented in Figs 10-12. With increase of turbulent mixing coefficient the initial part of the jet is shortened as well, $\varsigma_{i}=\kappa_{1} \bar{x}=0.86$ in figs, afterwards it is not real, because the initial part of the jet is finished, $y_{0}=0, \delta=\delta_{i}$. If turbulent mixing coefficient for water is 100 times higher than the laminar one, $\kappa_{1}=0.1$, then $\varsigma_{i}=\kappa_{1} \bar{x}=0.86$ means $\bar{x}=8.6$, so that the initial part of the jet is just 8.6 radiuses of the jet by $i_{0}=1.2, \kappa_{21}=1.0$ :
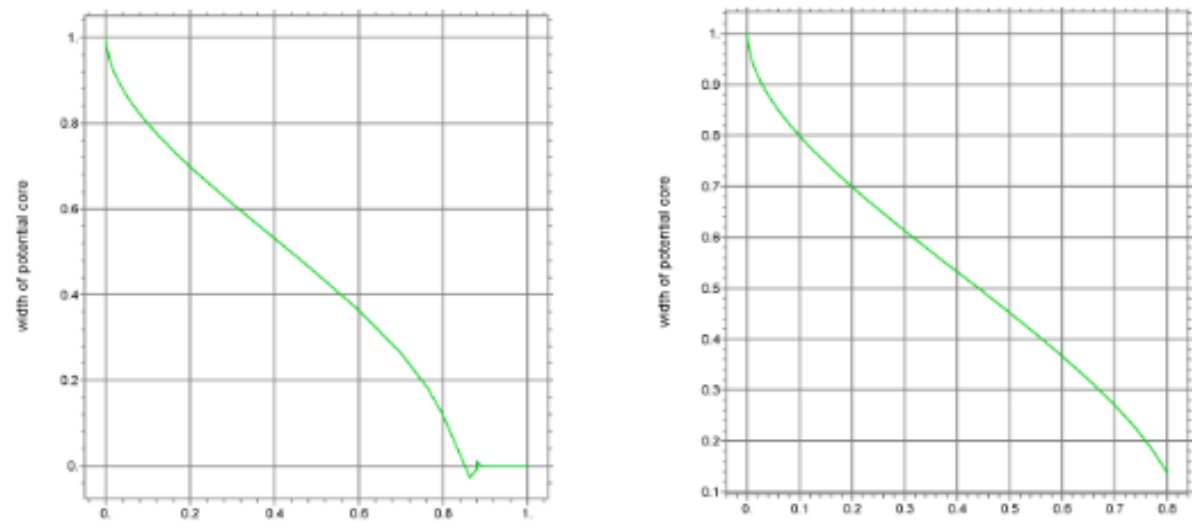

Fig10. The width of potential core $y_{0}(\zeta)$ in two different scales for parameters: $i_{0}=1.2, \kappa_{21}=1.0$
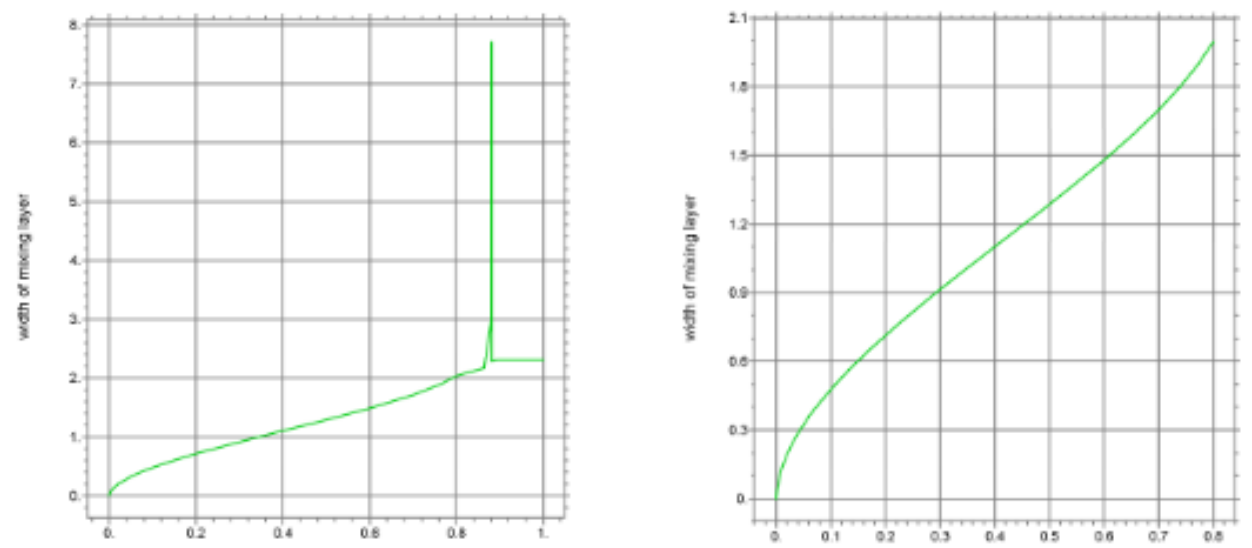

Fig11. The width of mixing layer $\delta(\zeta)$ in two different scales for parameters: $i_{0}=1.2, \kappa_{21}=1.0$
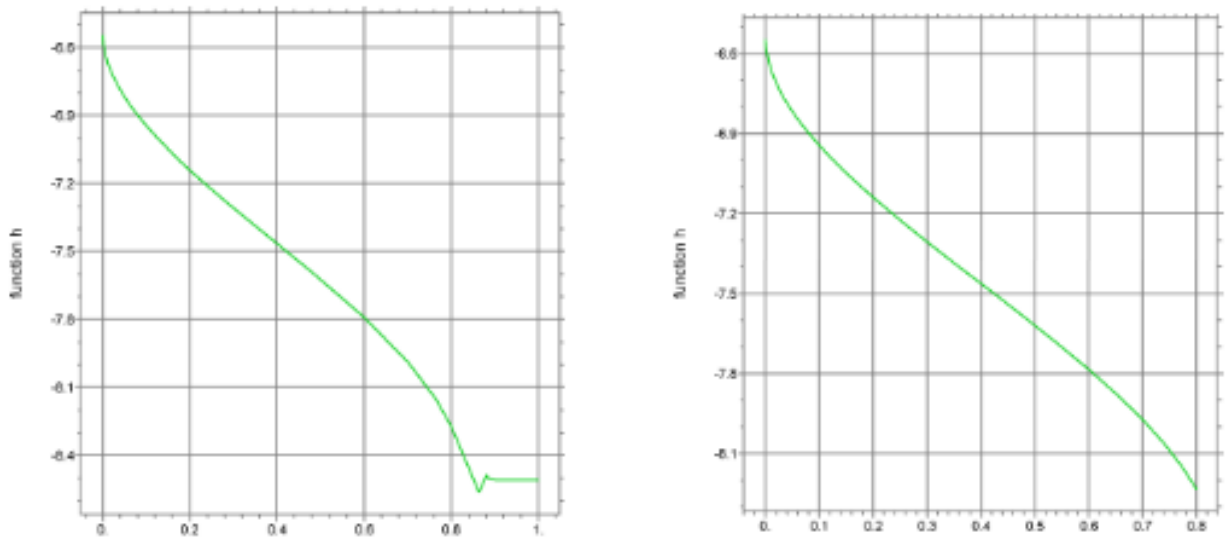

Fig12. The function $h(\zeta)$ in two different scales for parameters: $i_{0}=1.2, \kappa_{21}=1.0$

As shown in Figs 1-12 above, the length of the potential core and the width of the mixing layer depend on the density ratio and turbulent mixing coefficient. The calculations allow choosing the right regime and optimal characteristics in each specific case. Also the function $h$ is used to determine the phase distribution across the mixing layer, which is important for analysis of the mixing processes. This paper continues the previously done modeling of peculiarities of turbulent heterogeneous jets of mutually immiscible liquids [1,7]. 


\section{THE CONCLUSION}

The initial part of the turbulent heterogeneous two-phase jet of two mutually immiscible liquids was numerically simulated. Two cases were considered: jet of water spreading in the pool of oil and vise versa jet of oil spreading in the pool of water. A few different available turbulent mixing coefficients have been chosen for modeling. In petroleum business, it may be useful for precise description of the mixing process including phase distribution, which can be the new valuable opportunity for technical estimations and analysis.

\section{ACKNOWLEDGEMENT}

The author devotes this article to the blessed memory of Prof. Alfred I. Nakorchevskii. And the author wishes to acknowledge Prof. Torsten H. Fransson for the possibility to work at the Dept of Energy Technology, Royal Institute of Technology (KTH), during many years since 2001.

\section{REFERENCES}

[1] Nakorchevski A.I. Heterogeneous turbulent jets. Kyiv: Naukova Dumka, 1980, 142 p.

[2] Ginevskii A.S. The theory of turbulent jets and traces. Integral calculation methods. Moscow: Mashinostroenie, 1969, $400 \mathrm{p}$.

[3] Nakoryakov V.E., Pokusaev B.G., Shreiber I.R. Spreading of the waves in gas- and vapor-liquid media. Novosibirsk: ITF, 1983, 238 p. (In Russian).

[4] Nigmatulin R.I. Fundamentals of mechanics of heterogeneous media. Moscow: Nauka, 1978. - 336 c. (In Russian).

[5] R.I. Nigmatulin and John C. Friedly. Dynamics of Multiphase Media. CRC Press: Volumes 1 \& 2. Revised and Augmented Edition (v. 1 \& v. 2), Oct 1, 1990, 878 p.

[6] Nikolay Ivanov Kolev, Multiphase Flow Dynamics 1. 2015. Springer International Publishing. 840 p. (totally 5 volumes by different applications including thermo hydraulics in nuclear energy processes).

[7] Kazachkov Ivan V. Mathematical Modeling of the Mixing and Heat Transfer in Turbulent Two-Phase Jets of Mutually Immiscible Liquids// WSEAS Transactions on Heat and Mass Transfer, 2020, 15 (16), p. 117 129.

Citation: Ivan V. Kazachkov, "Modeling of the Turbulent Oil-Water Mixing Processes", International Journal of Petroleum and Petrochemical Engineering, 6(3), pp.38-45. DOI: https:// doi.org/10.20431/24547980.0603005

Copyright: (C) 2020 Authors, this is an open-access article distributed under the terms of the Creative Commons Attribution License, which permits unrestricted use, distribution, and reproduction in any medium, provided the original author and source are credited. 